\title{
Integrating Blockchain and Broad Learning for Smart Energy Innovation: Design and Experiment
}

\author{
Yubo Zhai ${ }^{1,2,{ }^{*}}$, Xianghan Zheng ${ }^{1,2,4}$ and Songpu $\mathrm{Ai}^{3,4}$ \\ ${ }^{1}$ College of Mathematics and Computer Science, Fuzhou University, Fuzhou 350116, \\ China \\ ${ }^{2}$ Fujian Key Laboratory of Network Computing and Intelligent Information \\ Processing, Fuzhou 350116, China \\ ${ }^{3}$ Research Institute of Information Technology, Tsinghua University, Beijing 100084, \\ China \\ ${ }^{4}$ Blockchain Innovation Lab, MingByte Technology (Qingdao) Co., Ltd., Qingdao \\ 266041, China \\ *Corresponding author e-mail: 1115373971@qq.com
}

\begin{abstract}
With the rapid development in recent years, the architecture and data sources of energy internet (EI) becomes increasingly complicated. It is a good opportunity and trend to combine energy equipment and information based on blockchain technology. However, high computational complexity inherently due to the blockchain must be addressed to achieve rapidly user electricity prediction. In this paper, an algorithm model of a blockchain technology combined with a broad learning data fusion algorithm is proposed. A novel and detailed combination model is introduced, mainly including CNN and LSTM model. Combination model considers energy data and blockchain data as parameter, and defines selection logic to guarantee user electricity forecast accuracy. Our proposed solution takes the advantage of CNN model in prediction accuracy and computation complexity, and advantage of LSTM model in long term memory. Further experiments illustrate that our solution achieves higher prediction accuracy of $94.1 \%$ (without considering the influence of real-time data), with the best result achieved ever. The experimental prediction results provide underlying insights to guide the direction of system resource planning.
\end{abstract}

KEYWORDS: Blockchain, Broad Learning, CNN+LSTM, Data Fusion, Energy Internet. 
Academic Journal of Computing \& Information Science

ISSN 2616-5775 Vol. 3, Issue 1: 59-77, DOI: 10.25236/AJCIS.030107

\section{Introduction}

Energy internet (EI) is an energy information fusion network constructed by the Internet concept, with a large-power grid as the backbone network and a micro-grid as the local area network [1]. EI is a kind of pan-energy system. Under the guidance of new connotations such as open and interconnected, user-centered and distributed peer-to-peer sharing, its energy transactions will tend to be diversified, the information will be transparent, and transactions will be timely [2]. EI has an open and peer-to-peer information integration architecture, and truly realizes multidirectional on-demand transmission of energy and dynamic balanced applications [3]. There is a trend of "three flows" of energy flow, information flow and value flow. Many countries in the world have started the research and development of their own EI projects. The FREEDM research project in the United States has proposed the concept of the Internet of Energy from the perspective of distributed power distribution network [4]. The Swiss Future Energy Network Vision project focuses on the integration of different energy forms, in line with RIFKIN J's concept of multi-network integration [5]. The E-Energy plan in Germany proposes the concept of the Internet of Energy from the perspective of ICT (the information and communication technology) to optimize the operation of power systems [6].

EI faces multiple obstacles at the technical and institutional levels. For example, the operation decision of a complex system, the breadth balance of multiple energy sources, the credibility of the open network subject, and the trading mechanism of a shared platform. Under the current model, energy transactions require a large number of third-party management agencies to build and maintain transaction credits, and therefore generate unnecessary high costs [7]. At the same time, the complexity of data information, data sources, and related information in the EI continues to increase. The multi-source observation information stored in the EI Data Center cannot be merely a simple and continuo us accumulation but need to be effectively fused and processed. Due to the development of data information, the development of information capacity and information processing speed have far exceeded the processing capacity of previous systems. To promote the further development of the energy system in the EI, the existing energy trading model needs to be changed, and the data fusion technology in EI needs to be updated.

The decentralization, transparency, and fairness of blockchain technology are in line with the concept of EI [8]. The application of blockchain technology in the energy network will further promote the construction and change of EI [9]. The stages of blockchain technology [10] research can be divided into the first stage with "Bitcoin [11]" as main technology, the second stage with "Ethereum [12]" as main technology, and the third stage with "HyperLedger [13]" as main technology. The current stage is in the period from the second phase to the third phase of the blockchain research. The in-depth study of blockchain and the diversified presentation of data make the third-stage application areas of blockchain continue to expand (for example, data tracking, anti-counterfeiting traceability, identity authentication, intellectual property protection, energy manage [14]). 
Academic Journal of Computing \& Information Science

ISSN 2616-5775 Vol. 3, Issue 1: 59-77, DOI: 10.25236/AJCIS.030107

At present, many companies and researchers have begun to explore the application of blockchain technology in EI. The Filament Company in the United States experimentally arranged a set of detection devices called "taps" on the grid nodes in the outback of Australia, and established a corresponding communication mechanism for these detection devices based on the blockchain system [15]. The US energy company LO3 Energy, in collaboration with Consensus Systems, a Bitcoin development company, has established an interactive grid platform based on the blockchain system called 'TransActive Grid', in the Gowanus and Park Slope neighborhoods of Brooklyn, New York [16]. The Scanergy project in EU aims to achieve direct transactions of small users' green energy based on blockchain systems [17].

In the field of research, there have been some successful applications of blockchain to deal with EI. In [18], scholars have proposed a mechanism framework for implementing distributed decision-making and coordinated autonomy of EI multi-module system using blockchain technology, and discussed the key technologies of blockchain and EI. In [19], scholars have proposed an automatic demand response scheme for a decentralized energy storage system based on the blockchain. The corresponding subjects in EI will automatically adjust their power requirements according to the system's real-time operating conditions and parameters according to established criteria. In [20], scholars have proposed a method of weakly centralized management of power transactions based on blockchain in the EI, which enables market participants to spontaneously trade based on smart contracts.

The related research and company projects introduced above have solved certain problems from different perspectives of the EI, but they have not achieved true multi-source data fusion applications. The combined apply of EI and Blockchain technology in this paper requires researchers to be able to effectively analyze and process data information in EI and Blockchain. There are three types of blockchainrelated data: intra-chain block data, off-chain related data, and inter-chain migration data. The block data in the chain records all transactions of the entire blockchain. The off-chain related data is the external correlation and supplement of the block data in the chain. In the research of this paper, it corresponds to the relevant power data information of the EI. Inter-chain migration data is interaction data between different blockchains. Research on data modeling and fusion based on these three types of data is helpful to analyze the behavior characteristics of each core element of the blockchain system.

Data fusion in the brand-new field of using blockchain technology, experts have launched research and exploration, but lack a solution supported by feature learning [21] theory as a tool, and the overall research is still in its infancy state. The work of this paper focuses on applying the algorithm model of broad learning in the EI system based on blockchain technology, and performing related manage such as fusion manage and processing analysis of multi-source data.

Broad learning [22] is a method of researching data analysis, which is used to perform fusion processing and mining on data from different data sources. Broad 
Academic Journal of Computing \& Information Science

ISSN 2616-5775 Vol. 3, Issue 1: 59-77, DOI: 10.25236/AJCIS.030107

learning is seen as an extension of transfer learning [23], which fuses data from different domains with complex network relationships to perform collaborative data mining and analysis. The core of data analysis algorithms based on broad learning have two characteristics: information fusion and knowledge discovery. The process of algorithm is divided into two steps: information fusion [24] and knowledge discovery. First, determine the information source of the problem, design the corresponding model which used to effectively fuse the information in the information source. Then perform deep mining and analysis of the fused heterogeneous data.

In the application of EI, broad learning can fuse the information (from various information sources inside and outside the power system) needed for the development of the energy system. The broad learning algorithm uses different energy data sources to extract useful information that can give guidance to datadriven EI business activities and use it as input to an automated decision-making model. Broad learning technology can effectively expand the source of massive data required for data driving, and improve the reliability of the generated decisions [25].

The algorithm in the paper takes $P O I$ features and user-related text information as inputs. Combining CNN and LSTM deep neural network for modeling, a contextual content awareness-POI prediction recommendation model [26] based on broad learning is applied to optimize and innovate the operation mode of EI. Energy equipment information, information data in the blockchain, and the evaluation of electrical energy users are integrated in the model to predict the electricity demand of electrical energy users in EI.

\section{Energy Internet Project}

\subsection{Project Introduction}

In recent years, State Grid has launched the "Ubiquitous Electricity Internet of Things [27]". This project based on the construction of the ubiquitous electric power Internet of Things aims to promote the deep integration of the power grid and the Internet, which comprehensively uses big data, blockchain technology, and new artificial intelligence [28] technology. "Ubiquitous Electricity Internet of Things" has fostered the development of new kinetic energy industries, expanded open sharing, created an EI ecosystem [29] and continued to promote innovation in energy production and consumption.

In traditional power grid organizations, substations, power stations, cables, user information, materials, and other resources are the basic elements that make up the ubiquitous electric power Internet of Things [30]. If data information of all elements is directly stored in the system data center without packaging, it will cause data management difficulties, poor scalability, poor interactivity, and poor availability. At the same time, the overall effectiveness of the ubiquitous electric power Internet of Things will be weakened fundamentally, which is unconducive to the unified platform to provide "differential" data services to the application layer. 
Academic Journal of Computing \& Information Science

ISSN 2616-5775 Vol. 3, Issue 1: 59-77, DOI: 10.25236/AJCIS.030107

The data sources of the power energy system [31] in this paper include the DMS database system, microgrid group management system, flexible demand side management system and $D C$ power energy management system. On-column switches, switch cabinets, and transformers make up DMS database system. Wind power generators, photovoltaic power generation equipment, and energy storage equipment form microgrid group management system. Flexible load-side management system consisting of industrial load, commercial load, residential load, electric vehicle. Build a multi-source data operation support platform based on the above four systems. Two modules of multi-source collaborative optimization control services and social public services are based on the data operation support platform (shown in Figure 1).

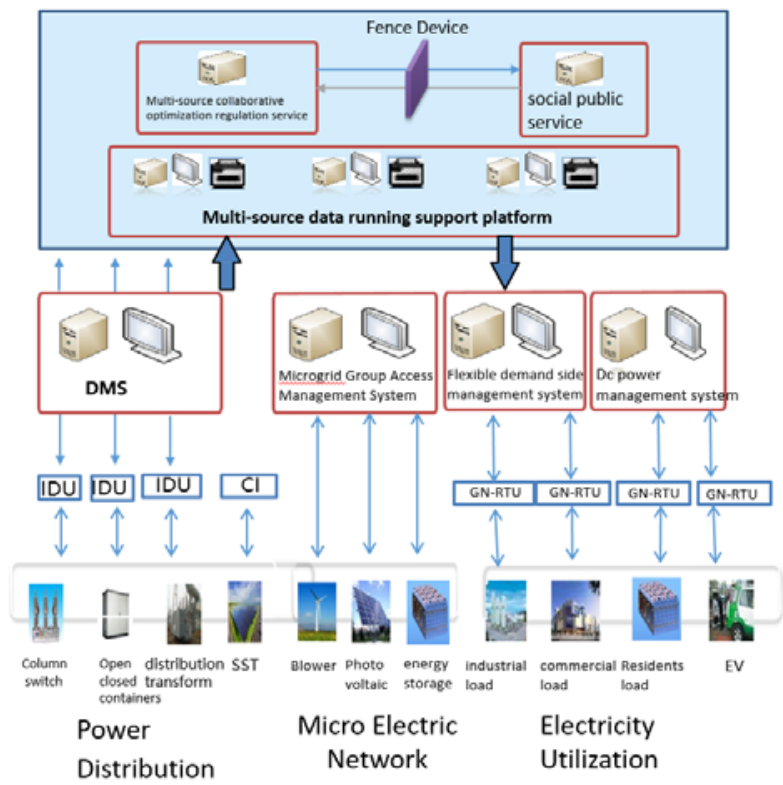

Figure 1. EI Project Architecture Diagram.

The data structure of the EI in the paper uses the "L-SRT" model [32]. This model is the logical top layer of "ubiquitous power IoT" and the logical principle of data abstraction for all realistic elements. The main constituent elements in a strong smart grid and the basic conceptual elements of the Internet are approximately functionally matched to each other. Abstract the elements of energy, materials, information, place, and transportation capacity according to their functions and attributes into one or more of L, S, R, and T. Abstract the people, money, things, and information in the electric power IoT into the "data" of the conceptual structure model. Construct a basic paradigm of data abstraction, so that the full-service data center at the platform layer can quickly allocate resources in the form of data according to application requirements. 
Paper proposes to build an energy information management platform based on blockchain technology. This design method has the data characteristics of multiple subject access and panoramic data access (shown in Figure 2).

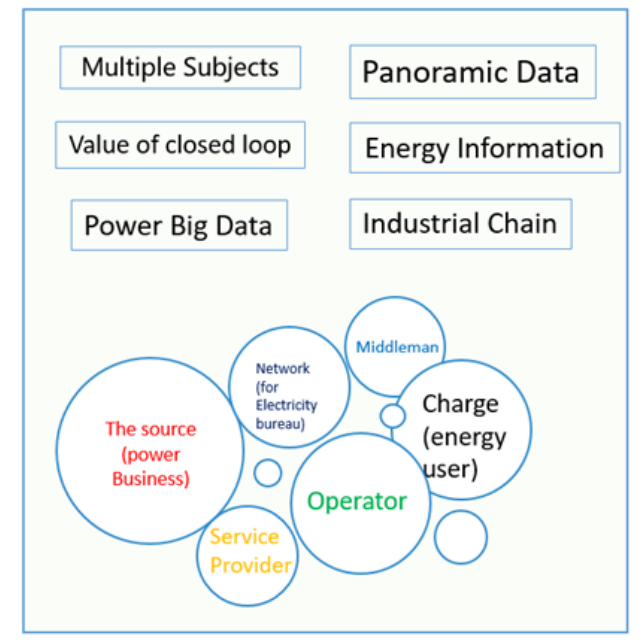

Figure 2. EI features.

EI based on block chain technology is a huge network system, including internal and external information from the power network system. Functions such as data collection, data integration, data storage processing, data services, and data applications have been implemented to support the stable operation of EI. The data source layer has multi-source data characteristics and is divided into internal data and external data to help data collection and processing. The data integration layer provides powerful data extraction, transformation, and loading capabilities for data analysis and use. The data storage function is divided into traditional database storage and distributed database storage, providing low-cost and highly scalable data storage solutions. The data storage functional layer can support storage requirements such as structured data, unstructured data, and semi-structured data; it can support low-latency and timely queries of structured and semi-structured data, and it can efficiently load and process unstructured data in batches. The data service layer provides cross-business analysis models and data mining algorithms; establishes a big data association analysis model library and algorithm library; implements flexible configuration and expansion of data analysis models and algorithms, and adds accurate real-time data value.

The combination of blockchain technology and energy routers promotes the integration of energy flow, information flow, and value flow, forms a closed loop of value and builds a next-generation energy infrastructure. Effectively fusing these multi-source and heterogeneous related data can mine more hidden knowledge to obtain more accurate prediction results. 
Academic Journal of Computing \& Information Science

ISSN 2616-5775 Vol. 3, Issue 1: 59-77, DOI: 10.25236/AJCIS.030107

Through the establishment of EI Analysis and Application Platform, various data of energy supply, consumption, and related technologies are integrated. This platform can provide big data analysis [33] and information services for government, enterprises, schools, residents and other different power users. EI-blockchain sharing platform provides users with personalized recommendations for power sales programs, popular power sales program planning, and other services. In this application, grid companies are the data provider of the integrated service platform, because have advantages in terms of capital, technology, and data resources.

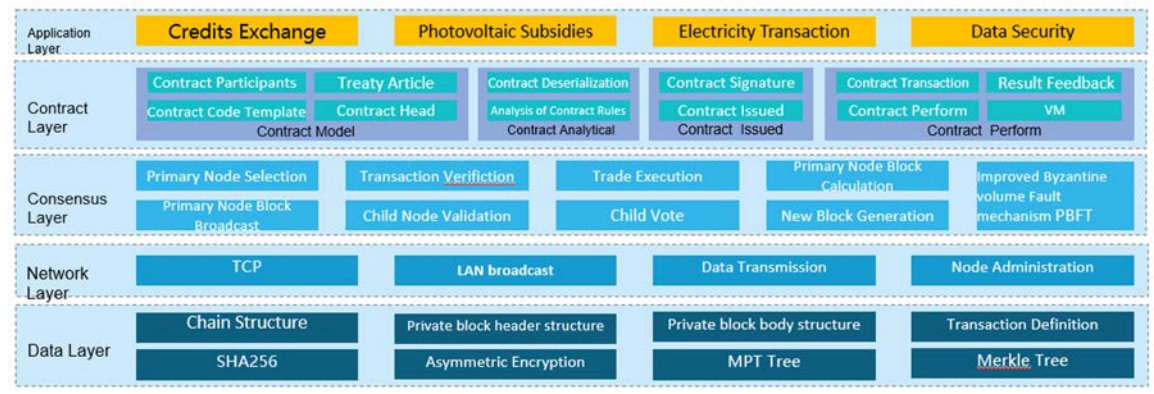

Figure 3. Application Architecture Diagram of Blockchain System.

\section{Experiment and methods}

\subsection{Data Fetch}

Paper's research project is based on blockchain technology to apply and manage EI projects. The data source of the experiment is blockchain transaction data, energy equipment data, and application feedback evaluation of electric energy users. The following describes the methods of obtaining and implementing data of each party in turn:

Intra-chain transaction data of blockchain: extract data generated by the operation of the blockchain. The extracted data content includes Blockfile block data, Consensus Algorithm data, historyDB data, and stateDB data.

First, block data in the Blockfile is parsed into JSON files format. After parsing into JSON files, we use Spark [34] software for data processing. Due to the high complexity of the data structure of the JSON file, Spark cannot be directly read and calculated to obtain the ideal result. Use wholeTextFiles to read file and call relevant data processing function to convert JSON format with high data complexity into JSON format with single line storage. For example, call explode function to parse JSON data in the nested format, and then call count function to count the number of transactions. Finally, parsed data is passed to Scala, and the script is called to perform calculations on JSON file (shown in Figure 4). 


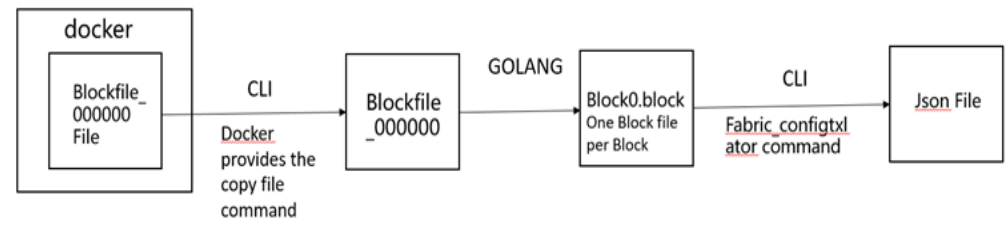

Figure 4. Block File Data Extraction Process.

The detailed data acquisition method is to call the data acquisition function (shown in Figure 5). The first step is to read blockfile binary file: write a for loop structure to extract the bytes array1 of each block, and then call destroyBlock() function to convert the bytes array1 to Common.Block type storage format. The second step is to call proto.Marshal() function to serialize common.Block type into an array of bytes array2, and then store the bytes array2 corresponding to each block into bytes.Buffer structure. The third step is to call deserializeBlock() function to convert data stored in bytes.Buffer into Json type and store Json files in the file system. Blockfile is composed of multiple serialized binary data. The binary data of each block consists of two parts: one part is a variable-length binary number with a maximum length of 8 bytes, representing the block length; the other is a binary number representing the content of the block.

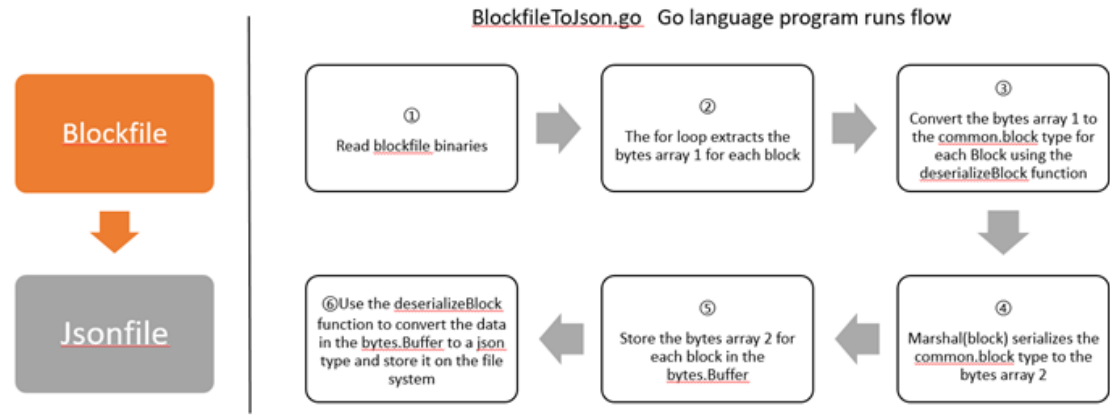

Figure 5. Block File Conversion to JSON File.

Data parsed in the block also needs to create RDD/DataFrame [35] for calculation. We store the data tuples describing blockchain into DataFrame data format. Use Scala [36] to create a List container to hold tuple data. Iterate through each transaction in blockfile, store parsed transaction data into the created tuple, and finally convert tuple into DataFrame format. Each row of DataFrame data format represents a transaction, and each column represents various attributes of transaction (shown in Figure 6). 


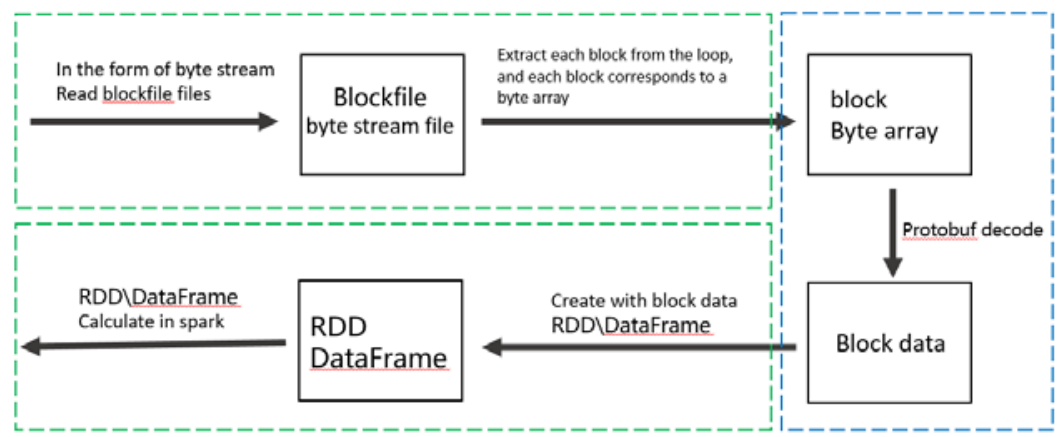

Figure 6. Data Format Conversion.

Experimental data also requires the number of calls of each chain code in blockchain, the number of transactions of each organization, the number of calls of each chain code, and the number of read and write sets. After getting all the data, you can call groupBy() function to group columns of the Dataframe to achieve statistical analysis. "Timestamp" records the number of transactions that occurred within the time stamp, "Block number" records the number of transactions contained in block, "Function name" records the number of times chain code function is called, and "Mspid" records "MSP"(Management Service Providers) submitted the number of transactions, "Chaincode name" records the number of times chain code was called.

Relevant data of kafka [37] consensus algorithm based blockchain is also a part of experimental data. In the docker [38] container of blockchain, call "Kafka consumer script" to obtain consumer data and create data as a KafkaInputDStream object. Parameter of KafkaInputDStream object is the IP field of Zookeeper [39] and Topic name is connected. KafkaInputDStream object can be connected to Kafka to decode the corresponding topic data. Kafka data can be parsed into three types: KafkaMessageRegular (message type generated by sorting), KafkaMessageTimeToCut (message type generated by packaging into block processes) and KafkaMessageConnect (message type generated by the connection process). Kafka data is a two-tuple of the form $\langle K, V\rangle$, and the data structure of $\langle K$, $V>$ is a String type. $V$ obtains byte array by calling the getbytes() function, and then deserializes byte array by calling the protobuf() function. Both HistoryDB data and StateDB data of blockchain can be obtained from Blockfile block data and do not need to be obtained again.

Energy equipment data and user feedback comment data belong to the off-chain data part of the blockchain. Energy equipment data is obtained and recorded in realtime through sensors of smart devices. In this study, energy equipment data were classified into four different data feature groups. "Operations" stands for business operation data for energy equipment. "State codes" represents the status codes of energy devices. "Hwinforegs" represents the hardware usage of the device. 
"Commands" stands for the marking instructions for energy equipment. "Regcategories" represents basic information about power equipment. Smart energy data structure is shown in Figure 7.

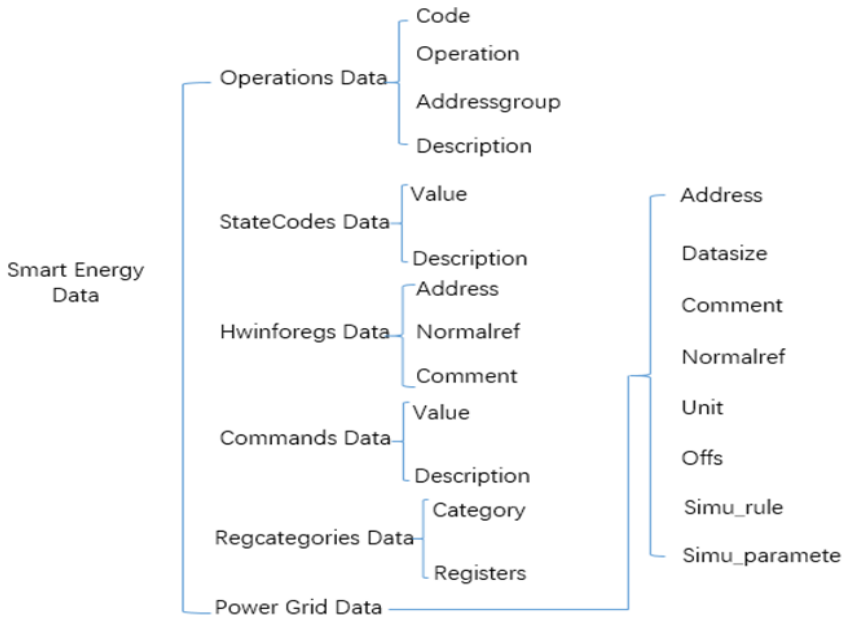

Figure 7. Smart Energy Data Set.

\subsection{Methods}

The project of the paper takes HyperLedger [40] blockchain technology as the underlying technology, combined with Hadoop [41] big data application technology. Use Apache Flume to store data in HDFS [42], and finally use Spark to obtain data in $H D F S$ for calculation (shown in Figure 8).

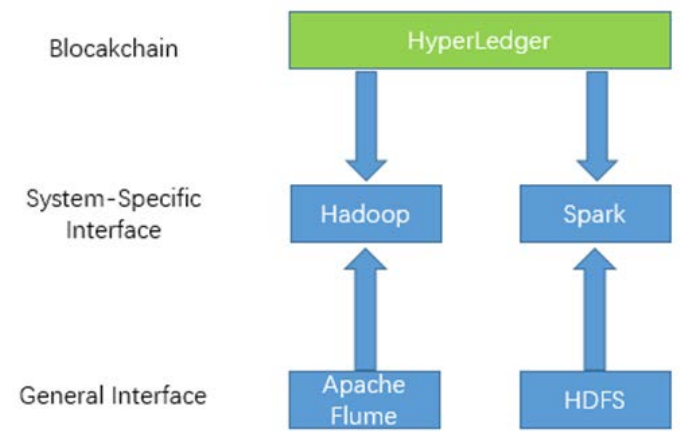

Figure 8. Architecture of Project.

A combined model for broad learning in this project-CNN and LSTM fusion model (shown in Figure 9).

Published by Francis Academic Press, UK 


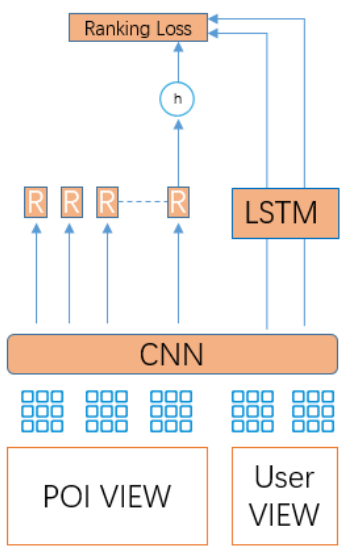

Figure 9. Data Fusion Breadth Learning Algorithm Model.

The combined model maps multiple types of data (data block data, energy equipment perspective, user perspective) to high-dimensional non-linear potential space (POI space), and then uses word embedding technology to represent data as a vector of low-dimensional space. Based on this, a simple, preliminary distributed representation is formed as the input to the model.

Assuming the dimension of the vector is $d$, the length of the comment sentence from the POI perspective is $l_{P}$, and the length of the evaluation sentence from the user perspective is $l_{u}$, then each comment in the POI perspective is recorded as $X^{p} \in R^{l_{p} \times d}$, shown in formula(1). Each comment from the user perspective is recorded as $X^{u} \in R^{l_{u} \times d}$, shown in formula(2).

$$
\begin{aligned}
& X^{p}=\left\{X_{1}^{P}, X_{2}^{P}, \ldots, X_{1}^{P}\right\} \\
& X^{u}=\left\{X_{1}^{u}, X_{2}^{u}, \ldots, X_{1}^{u}\right\}
\end{aligned}
$$

Sum $x_{i}^{p}$ and $x_{i}^{u}$ is sub-vector of the $i$ position of sum $X^{P}$ and $X^{U}$. Using $X^{P}$ and $X^{U}$ as inputs to a convolutional neural network model ( $C N N$ [43]), abstract local features are extracted through a convolution operation. For example, the concatenation of the $i$ to $j$ sub-vector in an energy data is $X_{i: j}$, as shown in formula (3). The length of the convolution kernel is $h$ and the weight matrix of the convolution kernel is $\mathrm{W} \in R^{h * d}$, convolution operation process is shown in formula (4). The abstract features extracted by convolution operation $C$, shown in formula (5). $L$ is the length of a data vector, $\mathrm{b} \in R^{d}$ is the offset term.

$$
\begin{aligned}
& X_{i: j}=x_{i} \oplus x_{i+1} \oplus \ldots \oplus x_{j} \\
& c_{i}=f\left(W^{\circ} X_{i: i t h-1}+b\right) c_{i}
\end{aligned}
$$




$$
c=\left[c_{1}, c_{2}, \ldots, c_{L-h+1}\right]
$$

Let $X^{P}$ and $X^{U}$ be the features obtained after $C N N$ training as $C^{P}$ and $C^{U}$ respectively. The characteristics $C^{U}$ of user perspective are not transformed, and the characteristics $C^{U}$ of the behavioral perspective are used as input to LSTM. [44] (long-term and short-term memory neural network).

Output $\mathrm{h}$ at the last moment in LSTM is the final feature of the behavioral perspective. Semantic sequence features of data text can be learned in LSTM. Each block cell depends on output $h_{t-1}$ of hidden layer at previous moment and input $x_{t}$ at current moment, $t$ represents the current moment and $t-1$ represents the previous moment. Each block contains three thresholds and a memory cell state at the current moment. The input threshold is $i_{t}$, the output threshold is $O_{t}$, the forget threshold is $f_{t}$ and the current cell state is $C_{t}$, Principles of $L S T M$ are shown in formula(6-11).

$$
\begin{gathered}
i_{t}=\sigma\left(W^{i} x_{t}+U^{i} h_{t-1}+b^{i}\right) \\
f_{t}=\sigma\left(W^{f} x_{t}+U^{f} h_{t-1}+b^{f}\right) \\
o_{t}=\sigma\left(W^{o} x_{t}+U^{o} h_{t-1}+b^{o}\right) \\
u_{t}=\tanh \left(W^{u} x_{t}+U^{u} h_{t-1}+b^{u}\right) \\
c_{t}=i_{t}{ }^{o} u_{t}+f^{o} c_{t} \\
h_{t}=o_{t}^{o} \tanh \left(c_{t}\right)
\end{gathered}
$$

Values of $O_{t}, f_{t}$ and $i_{t}$ are in the range of [0,1], $h_{t}$ is the output of the hidden layer at the current time $t, W^{i}$ and $U^{i}$ represent the weight matrix to be trained, $\delta$ represent activation function, $f_{t}$ and $u_{t}$ represent the output value of activation function. Output $h$ at the last moment of LSTM is taken as the final feature of the POI perspective. Finally, the top-level trend prediction layer merges the potential representations $C^{p}$ and $h$ generated by the first two layers (convolution layer $C N N$, recursion layer $L S T M$ ), and sets the entire model architecture prediction purpose to minimize errors, $y \in\{0,1\}^{n_{\text {samples }} * n_{\text {labels }}}$ is real label of data and $L_{i j}=\left\{(k, l): f_{i k}<f_{i j}, y_{i k}=1, y_{i l}=0\right\}, f \in R^{n_{\text {samples }}{ }^{*} n_{\text {lobel }}}$ is prediction of classifier. As shown in formula (12):

$$
\operatorname{ranking}(y, f)=\frac{1}{n_{\text {samples }}} \sum_{i=1}^{n_{\text {sanples }}} \frac{1}{\left|y_{i}\right|\left(n_{\text {labels }}-\left|y_{i}\right|\right)}\left|L_{i j}\right|
$$

\section{Results and discussion}

The combined model predicts energy distribution, supply trend of EI platform and user's power demand by training on multiple data sets. And final prediction data is within the minimization error range defined by ranking function. The experimental training model gives the accuracy of prediction. According to the 
accuracy, it provides a better service supply for users in energy supply network. In this experimental data set, $85 \%$ of the data is used as training data and $15 \%$ of the data is used as test data. According to the final result set, the experimental evaluation indicators shown in Figure10 can be obtained:

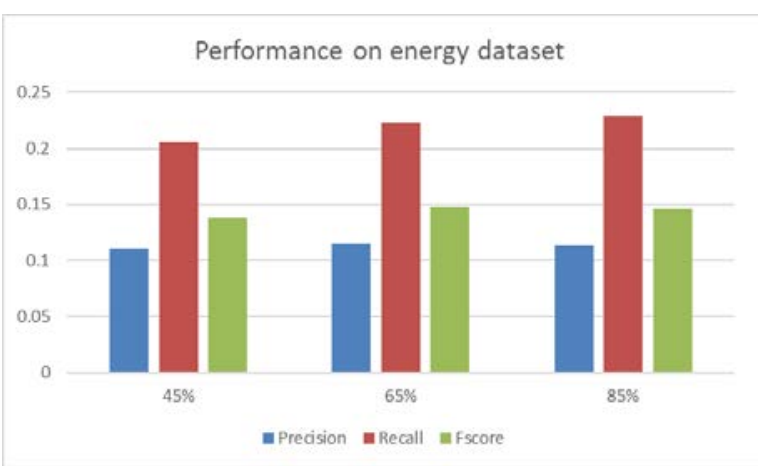

Figure 10. Data Training Distribution Ratio

Figure 10 shows the experimental results of the energy data set divided into a training set and test set according to different proportions. To verify the rationality of the design model of this paper, the Retailrocket dataset, Last.fm dataset and Energy dataset were used for testing. The Retailrocket data set is often used in the prediction work of the recommendation system. It collects data from a real-world ecommerce website. All values in the data set are hashed. The Last.fm dataset is also commonly used in recommendation systems. It is a dataset about the sequence of songs that users listen to. As a representative of the implicit feedback data set of context information, it includes two parts: listening to songs and user information. The commonly used public data sets are input into this model for verification, which increases the contrast of the experiment and also verifies the advantages of the model. Effect of the experimental dataset is shown in Figure 11-Figure 13.

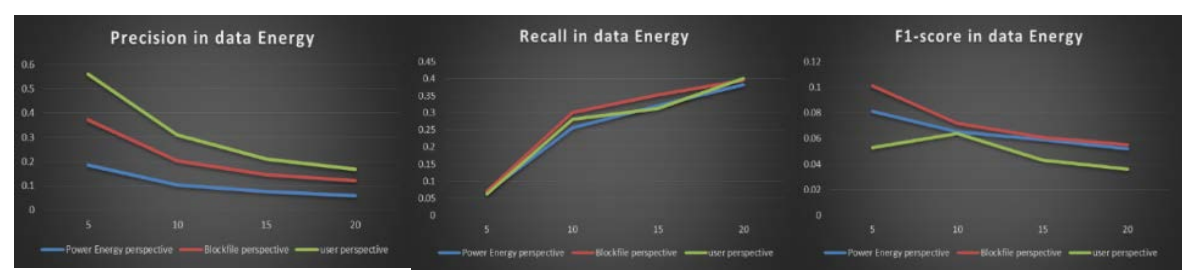

Figure 11. Effect of Energy Dataset. 


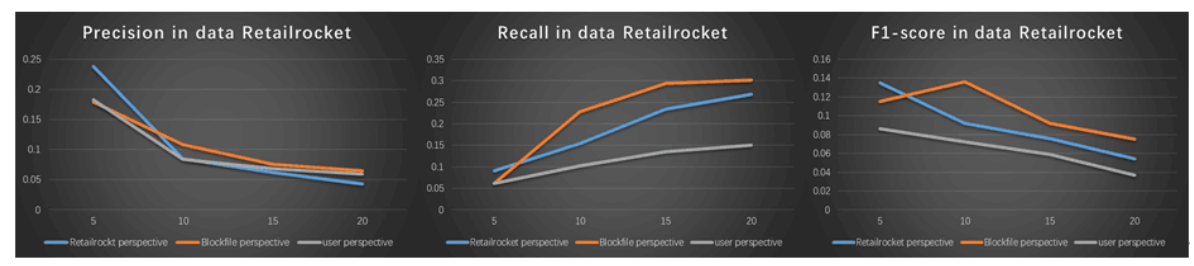

Figure 12. Effect of the Retailrocket Dataset.

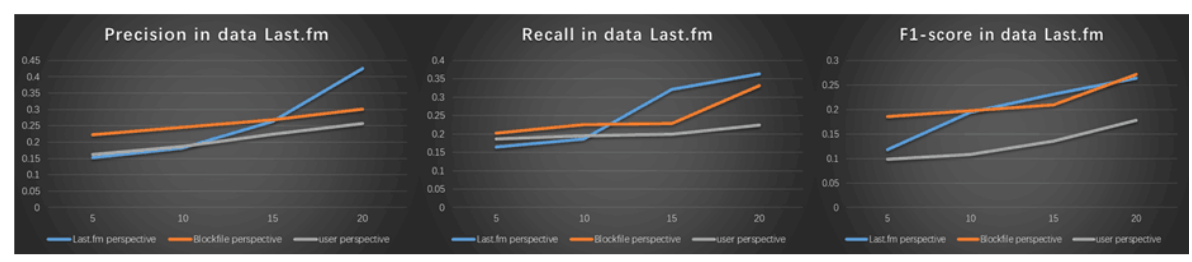

Figure 13. Effect of Last. Fm Dataset.

In the presentation of the experimental results of the energy dataset, three data perspectives: energy equipment data, block data, and user perspective data will all play different proportions in the prediction of the data results. Among the three different data sections, the energy equipment data has the largest effect on the prediction accuracy, and the block data has the largest impact on the prediction recall rate. In the experiments of the Retailrocket dataset and Last.fm dataset, the contribution of block data to the prediction accuracy is also the largest, which proves the correctness of building EI model based on blockchain technology. Detailed obfuscation matrix data are shown in Table 1:

Table 1 Different Data Set Confusion matrix evaluation.

\begin{tabular}{|c|c|c|c|}
\hline DataSet & Precision & Recall & F1-score \\
\hline Energy Data & 0.934 & 0.948 & 0.941 \\
\hline Retailrocket Data & 0.921 & 0.924 & 0.922 \\
\hline Last.fm Data & 0.893 & 0.908 & 0.901 \\
\hline
\end{tabular}

Testing experiments with different data sets is the first step of model detection, and comparing the experimental results of energy data sets in different algorithm models is the second step of combined model detection. The combination model is compared with the four algorithms selected by the traditional machine learning model library: Decision Tree Method, Random Forest, Logistic Regression, and $S V M$, and the four artificial neural network models selected by the neural network learning algorithm: $R B F$ [45], GMDH [46], $L M$ [47], FNN for detection and comparison. As can be seen from the data results in the Table $(2,3)$, the precision 
Academic Journal of Computing \& Information Science

ISSN 2616-5775 Vol. 3, Issue 1: 59-77, DOI: 10.25236/AJCIS.030107

value of the $C N N+L S T M$ combination model can reach 0.947 , the recall value is 0.936 , and the F1-score value is 0.941 . The effect value is better than each machine learning algorithm and each neural network learning algorithm, which validates the advantages of the combined model.

In addition to these optimistic data, we also need to note that the energy data set has only a low error in the training data in the detection of different models. However, this is not the case because of the lack of rigorous test data in the data set. For example, in traditional data models, a mistake is using a model with 1000+ parameters to fit a data set with only 200 observations. Another kind of undetectable error is: use a regression model to divide data set into parameter estimation and verification calculations, and these two parts determine the percentage error (Mean Absolute Percentage Error). When the calculated MAPE is too high, replace the model and recalculate MAPE until MAPE value is small enough. Although these validation data are not used for model fitting, they are used when constructing model variables. In reality, it is impossible to build models using exact future energy data. To sum up, you can get good results by glancing at future data, but it is impossible in practice. Detailed comparison diagram is as follows Figure 14-Figure 15:

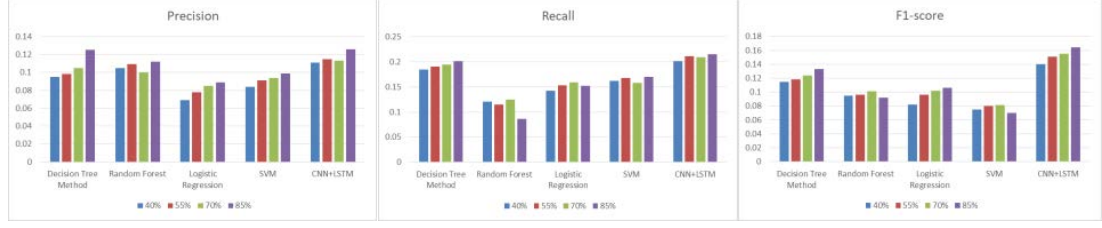

Figure 14. Results of Traditional Machine Learning Algorithms.

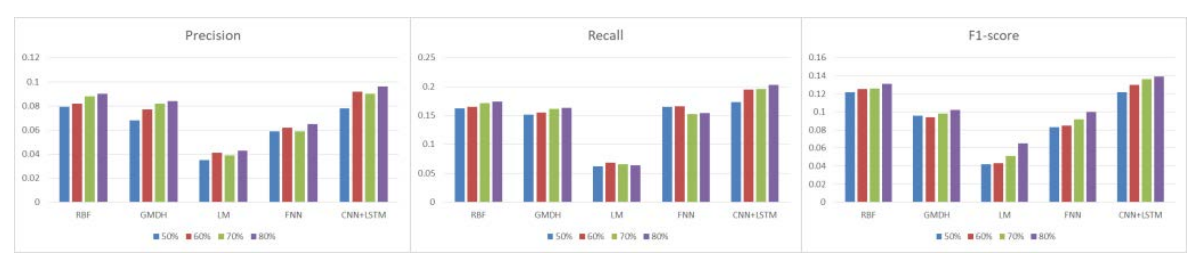

Figure 15. Results of Artificial Neural Network Algorithm.

Table 2 Different Traditional Machine Learning Confusion matrix evaluation.

\begin{tabular}{|c|c|c|c|}
\hline Method & Precision & Recall & F1-score \\
\hline Decision Tree & 0.842 & 0.829 & 0.835 \\
\hline Random Forest & 0.843 & 0.857 & 0.850 \\
\hline Logistic Regression & 0.882 & 0.861 & 0.871 \\
\hline SVM & 0.919 & 0.898 & 0.908 \\
\hline CNN+LSTM & 0.947 & 0.936 & 0.941 \\
\hline
\end{tabular}

Published by Francis Academic Press, UK

$-73-$ 
Academic Journal of Computing \& Information Science

ISSN 2616-5775 Vol. 3, Issue 1: 59-77, DOI: 10.25236/AJCIS.030107

Table 3 Different Artificial Neural Network Confusion matrix evaluation.

\begin{tabular}{|c|c|c|c|}
\hline Method & Precision & Recall & F1-score \\
\hline RBF & 0.832 & 0.845 & 0.838 \\
\hline GMDH & 0.851 & 0.834 & 0.842 \\
\hline LM & 0.857 & 0.887 & 0.872 \\
\hline FNN & 0.909 & 0.923 & 0.916 \\
\hline CNN+LSTM & 0.947 & 0.936 & 0.941 \\
\hline
\end{tabular}

\section{Conclusion}

Based on EI platform, this paper applies blockchain technology and broad learning to perform multi-source data fusion calculation, research. Paper comprehensively processes data of three modules of energy equipment data, blockchain data, and user feedback evaluation, and uses a combination of spark computing and deep learning for experiments. Through the prediction analysis of the experimental model, the user's demand for power and energy is given within specified minimum error range. In the end, information coordination of EI will be improved to provide users with better services.

In general, experimental results can effectively achieve the design goals of our experiments, and prediction accuracy is also within an acceptable range. We have conducted a series of experiments and analysis to show that our proposed $C N N+L S T M$ solution is feasible, efficient, and achieving a high prediction accuracy. Comparing with other algorithms or existing work, our proposed solution also contains acceptable computation complexity. We demonstrate our work for recent advances in blockchain and justify our research contributions to meet prediction challenges for EI. However, experimental research is carried out on the offline collected data set, the fusion analysis of real-time data has not been implemented for time being. This direction is one of the key points of future research.

\section{References}

[1] Daneshvar, Mohammadreza, Mahmoud Pesaran, and Behnam Mohammadiivatloo. "Transactive energy in future smart homes." The Energy Internet. Woodhead Publishing, 2019. 153-179.

[2] CAI jinqi, et al. Energy transactions based on block chain in energy Internet. Power Construction, 38.9 (2017) 24-31.

[3] Hou, Weigang, Lei Guo, and Zhaolong Ning. "Local Electricity Storage for Blockchain-based Energy Trading in Industrial Internet of Things."IEEE Transactions on Industrial Informatics15.6 (2019): 3610-3619.

[4] Huang, Alex Q., et al. "The future renewable electric energy delivery and management (FREEDM) system: the energy internet." Proceedings of the IEEE 99.1 (2010): 133-148. 
Academic Journal of Computing \& Information Science

ISSN 2616-5775 Vol. 3, Issue 1: 59-77, DOI: 10.25236/AJCIS.030107

[5] RIFKIN J. Third industrial revolution: how lateral power is transforming energy, the economy, and the world [M]. New York: Palgrave Macmillan Trade, 2011: 33-72.

[6] BLOCK C, BOMARIUS F, BRETSCHNEIDER P, et al. Internet of energy-ICT for energy markets of the future [R]. Berlin: BDI, 2010.

[7] ZHANG Jian, et al. "Research on Framework and Typical Application in Energy Internet” [J]. Proceeding of the CSEE, 2016, (15): 4011-4023.

[8] Zhang, Ning, et al. "Blockchain technique in the energy internet: preliminary research framework and typical applications." Proceedings of the CSEE 36.15 (2016): 4011-4022.

[9] S. Ai, C. Rong, and J. Cao, "Utilization of Big Data in Energy Internet Infrastructure,” in Energy Internet - Systems and Applications, 1st ed., Springer, 2020, ch. 9.

[10] Zheng, Zibin, et al. "Blockchain challenges and opportunities: A survey." International Journal of Web and Grid Services 14.4 (2018): 352-375.

[11] Nakamoto S. Bitcoin: A peer-to-peer electronic cash system[R]. Manubot, 2019.

[12] Wood, Gavin. "Ethereum: A secure decentralised generalised transaction ledger." Ethereum project yellow paper 151.2014 (2014): 1-32.

[13] Androulaki, Elli, et al. "Hyperledger fabric: a distributed operating system for permissioned blockchains." Proceedings of the Thirteenth EuroSys Conference. 2018.

[14] Pop, Claudia, et al. "Blockchain based decentralized management of demand response programs in smart energy grids." Sensors 18.1 (2018): 162.

[15] Tapscott, Don, and Alex Tapscott. "How blockchain technology can reinvent the power grid." 2016-05-15). http://fortune.com/2016/05/15/blockchainreinvents- power-grid (2016).

[16] Nguyen, Clinton. "An indie, off-the-grid, blockchain-traded solar power market comes to brooklyn." 2016-03-18). http://motherboard.vice.com/read/the-plan-topower-brooklyn-with-a-blockchain-based-microgrid-transactive-solar (2016).

[17] Prisco, Giulio. "An energy blockchain for European prosumers." Bitcoin Mag. https://bitcoinmagazine.Com/articles/an-energy-blockchain-for-europeanprosumers - 1462218142. Accessed 3 (2016).

[18] Zeng Ming, Cheng Jun, Wang Yuqing, et al. Research on multi module collaborative autonomous mode of energy internet based on blockchain framework [J]. Proceedings of the CSEE, 2017, 37 (13): 3672-3681 (in Chinese).

[19] Yang Xiaodong, Zhang Youbing, Lu Junjie et al. Automatic demand response of energy storage system in energy local area network based on blockchain technique [J] Proceedings of the CSEE, 2017, 37 (13): 3703-3716 (in Chinese).

[20] Tai Xue, Sun Hongbin, Guo Qinglai. Measures for power trading and congestion management based on blockchain in the energy internet [J]. Power System Technology, 2016, 40 (12): 3630-3638 (in Chinese).

[21] Grover, Aditya, and Jure Leskovec. "node2vec: Scalable feature learning for networks." Proceedings of the 22nd ACM SIGKDD international conference on Knowledge discovery and data mining. 2016. 
Academic Journal of Computing \& Information Science

ISSN 2616-5775 Vol. 3, Issue 1: 59-77, DOI: 10.25236/AJCIS.030107

[22] Chen, CL Philip, and Zhulin Liu. "Broad learning system: An effective and efficient incremental learning system without the need for deep architecture." IEEE transactions on neural networks and learning systems 29.1 (2017): 10-24.

[23] Yang, Qiang, et al. Transfer learning. Cambridge University Press, 2020.

[24] Mahler, Ronald PS. Statistical multisource-multitarget information fusion. Vol. 685. Norwood, MA: Artech House, 2007.

[25] Chen, CL Philip, Zhulin Liu, and Shuang Feng. "Universal approximation capability of broad learning system and its structural variations." IEEE transactions on neural networks and learning systems 30.4 (2018): 1191-1204.

[26] Wang P, Guo J, Lan Y, et al. Learning hierarchical representation model for nextbasket recommendation[C]/Proceedings of the 38th International ACM SIGIR conference on Research and Development in Information Retrieval. ACM, 2015: 403-412.

[27] Möller D P F, Vakilzadian H. Ubiquitous networks: Power line communication and Internet of things in smart home environments [C] //IEEE International Conference on Electro/Information Technology. IEEE, 2014: 596-601.

[28] Liu, Jiaying, et al. "Artificial intelligence in the 21st century." IEEE Access 6 (2018): 34403-34421.

[29] Sun, Yunchuan, et al. "Internet of things and big data analytics for smart and connected communities." IEEE access 4 (2016): 766-773.

[30] Dongsheng, Yang, et al. "Key technologies and application prospects of ubiquitous power Internet of Things." Power generation technology 40.2 (2019): 107-114.

[31] Ming, Yangyang, et al. "Distributed energy sharing in energy internet through distributed averaging." Tsinghua science and Technology 23.3 (2018): 233-242.

[32] Wang, Jiye, et al. "Electricity services based dependability model of power grid communication networking." Tsinghua Science and Technology 19.2 (2014): 121-132.

[33] Xue, Yusheng, and Yening Lai. "Integration of macro energy thinking and big data thinking part one big data and power big data." Automation of Electric Power Systems 40.1 (2016): 1-8.

[34] Armbrust, Michael, et al. "Spark sql: Relational data processing in spark." Proceedings of the 2015 ACM SIGMOD international conference on management of data. 2015.

[35] Flinsbaugh J W, Jones J, Mullendore R N, et al. High performance system providing selective merging of dataframe segments in hardware: U. S. Patent 9,304, 709 [P]. 2016-4-5.

[36] Odersky, Martin, Lex Spoon, and Bill Venners. Programming in scala. Artima Inc, 2008.

[37] Kreps, Jay, Neha Narkhede, and Jun Rao. "Kafka: A distributed messaging system for log processing." Proceedings of the NetDB. Vol. 11. 2011.

[38] Boettiger C. An introduction to Docker for reproducible research [J]. ACM SIGOPS Operating Systems Review, 2015, 49 (1): 71-79.

[39] Hunt, Patrick, et al. "ZooKeeper: Wait-free Coordination for Internet-scale Systems." USENIX annual technical conference. Vol. 8. No. 9. 2010.

Published by Francis Academic Press, UK

$-76-$ 
[40] Cachin, Christian. "Architecture of the hyperledger blockchain fabric." Workshop on distributed cryptocurrencies and consensus ledgers. Vol. 310. 2016 [41] Merkel, Dirk. "Docker: lightweight linux containers for consistent development and deployment." Linux journal 2014.239 (2014): 2.

[42] Borthakur, Dhruba. "HDFS architecture guide." Hadoop Apache Project 53.113 (2008): 2.

[43] Vaidyanathan S. 3-cells cellular neural network (CNN) attractor and its adaptive biological control [J]. International Journal of PharmTech Research, 2015, 8 (4): 632-640.

[44] Greff K, Srivastava R K, Koutník J, et al. LSTM: A search space odyssey [J]. IEEE transactions on neural networks and learning systems, 2016, 28 (10): 22222232.

[45] Liu, Jinkun. Radial Basis Function (RBF) neural network control for mechanical systems: design, analysis and Matlab simulation. Springer Science \& Business Media, 2013.

[46] Ivakhnenko, A. G., and G. A. Ivakhnenko. "The review of problems solvable by algorithms of the group method of data handling (GMDH)." Pattern Recognition and Image Analysis C/C of Raspoznavaniye Obrazov I Analiz Izobrazhenii 5 (1995): 527-535.

[47] Wilamowski, Bogdan M., and Hao Yu. "Improved computation for LevenbergMarquardt training." IEEE transactions on neural networks 21.6 (2010): 930-937. 